\title{
COVID-19: Notes From the Front Line, Singapore's Primary Health Care Perspective
}

\author{
Wei Han Lim, MBBS \\ Wei Mon Wong, MBBS, FCFP \\ Division of Primary Care, Raffles Medical \\ Group, Singapore
}

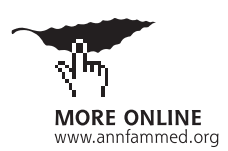

Conflicts of interest: authors report none.

\section{CORRESPONDING AUTHOR}

Dr Wei Han Lim

Raffles Medical Group

585 North Bridge Rd

Singapore 188770

lim_weihan@rafflesmedical.com

\begin{abstract}
Coronavirus disease 2019 (COVID-19) is a rapidly progressing global pandemic against which nations are struggling for containment. Singapore is known to have promptly instituted aggressive public health and containment measures. A key pillar sustaining this is the response of its primary health care network. It is important for health care systems worldwide to recognize the value of a strong coordinated response to this crisis from a primary health perspective. There are best practices for early isolation and containment of suspect cases while protecting health care workers and limiting cross infections that are transferable across nations. We describe our framework for how our primary care clinics respond to this pandemic in the hope others may find solutions to their unique needs. Moving forward, there is a pressing need for more studies to enhance our understanding of the response of primary care during these public health crises.
\end{abstract}

Ann Fam Med 2020;18:259-261. https://doi.org/10.1370/afm.2539.

\section{INTRODUCTION}

Coronavirus disease 2019 (COVID-19) has spread rapidly out of China, into greater Asia and now into Europe and America.

Singapore is particularly at risk due to its status as a global travel and business hub. It was among the first countries affected, confirming its first COVID-19 case on January 23, 2020. ${ }^{1}$ Singapore promptly instituted aggressive public health and containment measures, drawing from experience with the Severe Acute Respiratory Distress Syndrome (SARS) epidemic in 2003 and the Influenza H1N1 pandemic in 2009.

It is important for health care systems to recognize the value of a strong coordinated response to this crisis from a primary health perspective, ${ }^{2}$ with best practices that are transferable across nations. This is essential given that the early presentation of COVID-19 infection is nonspecific, with most patients presenting to primary health care clinics with mild upper respiratory tract symptoms.

Information and data available in the rapidly evolving body of literature on the management of COVID-19 in the primary health care setting is inadequate. Evidence for the best possible primary health care response during the $\mathrm{SARS}^{3}$ epidemic and the Influenza $\mathrm{H} 1 \mathrm{~N} 1^{4}$ pandemic is also suboptimal. This highlights the pressing need for more studies to enhance our understanding of the response of primary care during these public health crises. ${ }^{5}$ We describe our framework for how primary care clinics respond to this pandemic in the hope others may find transferrable solutions to their unique needs.

\section{PRIMARY HEALTH CARE RESPONSE IN SINGAPORE}

We share our experience with the implementation of containment measures from a network of 50 private general practitioner (GP) clinics, including containment and infection control strategies which are enhanced through government guidelines and logistical support. 
Singapore is heavily reliant on its primary health care network; GP clinics are the first point of contact for most undifferentiated cases, representing $79.06 \%$ of total primary health attendances in a day. ${ }^{6}$ During the pandemic, the clinics also must manage patients with nonrespiratory tract-related complaints. These patients need to be protected against the possibility of transmission of disease from positive cases during their visit. In addition, the safety of our health care workers must be secured with these containment measures, mitigating concerns and anxiety about personal safety $^{7}$ as a health care worker at the frontline, and maintaining a healthy workforce. Given the high volume of patient flow and the non-specific nature of early COVID-19 infection, GP clinics form the cornerstone of early identification and isolation of suspect cases within the vast patient pool.

As a network, during the period between February 8, 2020 and February 22, 2020, the clinics saw a total of 56,820 attendances. One hundred twenty-five were identified as suspect cases and sent to the National Centre for Infectious Diseases (NCID). Of these, 3 were tested positive. During that period, 1 clinic saw a total of 1,228 patients of which 13 patients were isolated, and 2 patients were identified as suspect cases and sent to NCID.

No health care worker within the network was infected with COVID-19 at the time of writing.

\section{Containment Measures}

Visits were streamlined following a fixed protocol within our network (Figure 1). Reception counter nurses triaged all patients to identify suspect cases following a strict protocol and a health declaration form (Supplemental Figure 1, http://www.AnnFamMed.org/
content/18/3/259/suppl/DC1) drawn from government guidelines regarding suspect case definition. These were updated according to the evolving situation locally and globally.

Once identified, the cases were immediately isolated in a designated room in the clinic for assessment by the doctor. If a patient fit the suspect case defini-

\section{Figure 1. Institution workflow for patients with respiratory tract symptoms.}

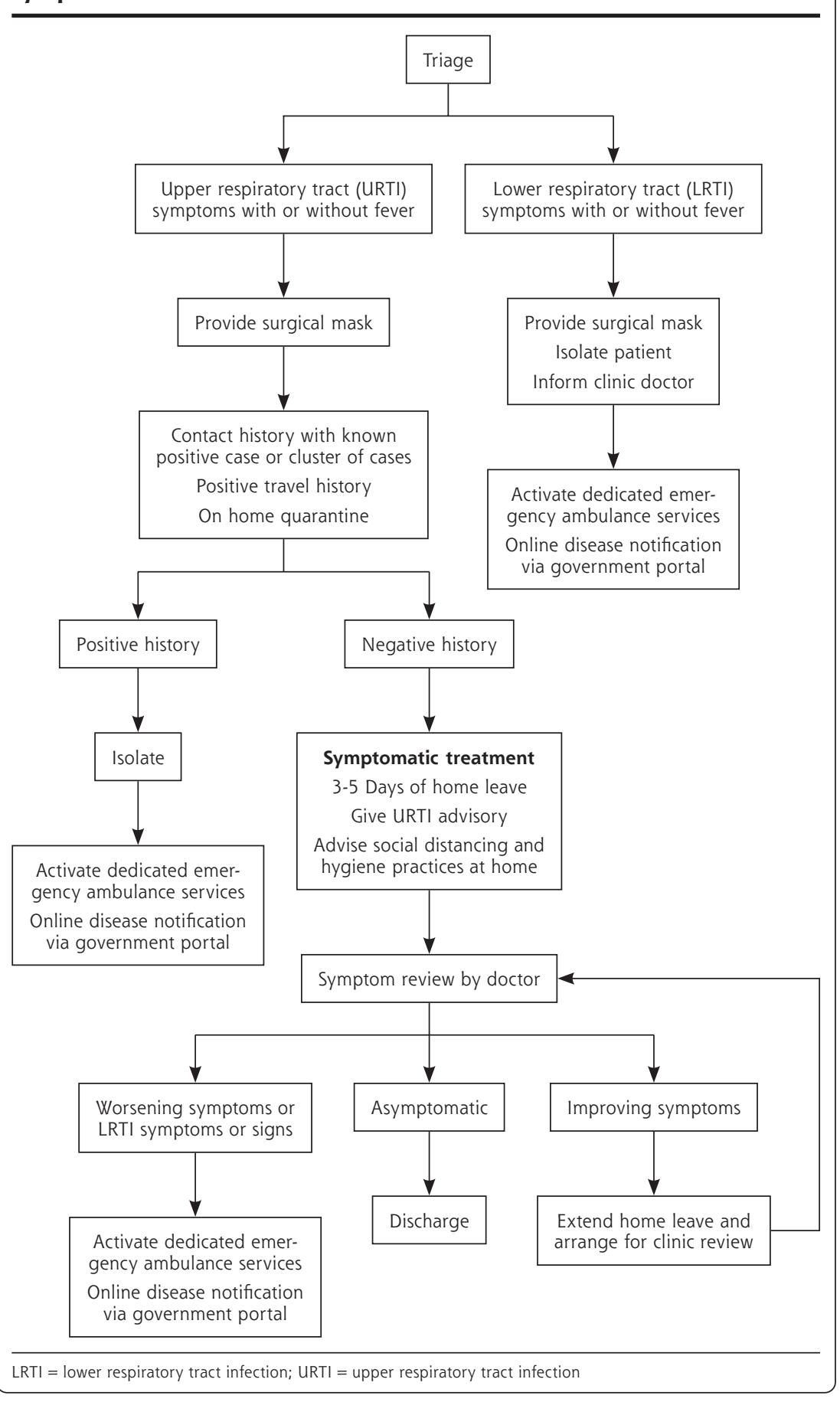


tion, this patient remained in isolation until evacuation for further evaluation by a dedicated public ambulance service to the NCID.

According to the natural progression of upper respiratory tract infections, nonsuspect cases were given 3 to 5 days of home medical leave and were highlighted for subsequent review via the group's electronic medical records (EMR) system. This enabled clinicians to track cases and allowed for longitudinal information flow within the network. Patients were then contacted by the clinic and reviewed via telephone call on the last day of their medical leave. Those who reported persistent symptoms were advised to visit the clinic for further workup while those who reported worsening symptoms such as breathlessness may have been brought from their home via the public ambulance service directly to the NCID for further evaluation. In the event a patient felt a progression of symptoms during the period of medical leave, they could either consult a doctor via the network's telemedicine mobile application or make a physical trip to the clinic for evaluation.

These measures worked in concert with government containment measures such as a mandatory 14-day stay-home restriction on returning travelers from high-risk regions of the world.

\section{Infection Control Measures}

Extensive infection control measures were put in place to prevent cross-contamination from potential positive cases to other patients and health care workers.

All medical staff within the network were promptly updated on new developments via multiple communication channels lke e-mail and secure mobile chat groups. This minimized difficulties with information access.

Appropriate training for health care workers was also enforced using one-on-one sessions and multimedia information guides. All medical staff were trained in the appropriate use and disposal of personal protective equipment (PPE). Doctors planned the management and disposition of patients according to the latest directives from the Ministry of Health while nurses were instructed in appropriate decontamination. This optimized the education of infection control measures at the level of the individual health care workers.

PPE resources were provided by both institution and government stockpiles with reassurance of adequate supplies for staff protection throughout the pandemic (Supplemental Figure 2). PPE was worn by reception staff for all patients at the counter, and by doctors during patient consultation - in both isolation room and normal consult room. Compliance to PPE guidelines was strictly enforced at all levels.
If a suspect case was identified and sent to NCID, the clinic would undergo subsequent decontamination prior to resuming services.

\section{CONCLUSION}

While hospitals and health systems are under tremendous strain during this pandemic, it is essential to bear in mind that prompt measures to contain and mitigate this should start at the grassroots level. These measures aim to identify and isolate cases early, reducing the burden of triage at hospital level, overcoming barriers of communication and education, while protecting our patients and health care workers. It is our hope that our experience may contribute towards a framework which other health care systems may adapt to their unique needs. Moving forward, we envision that our experience would prompt more widespread examination of the role of primary health care in pandemics such that globally, primary health care networks may mount prompt and effective evidence-based responses.

To read or post commentaries in response to this article, see it online at http:I/www.AnnFamMed.org/content/18/3/259.

Submitted March 16, 2020; submitted, revised, March 25, 2020; accepted March 25, 2020.

Key words: coronavirus; COVID-19; SARS-CoV-2; primary health care; virus; pandemic

Supplemental materials: Available at http://www.AnnFamMed. org/content/18/3/259/suppl/DC1/.

\section{References}

1. Ministry of Health, Singapore. Confirmed imported case of novel coronavirus infection in Singapore; multi-ministry taskforce ramps up precautionary measures. https://www.moh.gov.sg/newshighlights/details/confirmed-imported-case-of-novel-coronavirusinfection-in-singapore-multi-ministry-taskforce-ramps-up-precautionarymeasures. Published Jan 23, 2020. Accessed Feb 25, 2020

2. Kunin M, Engelhard D, Piterman L, Thomas S. Response of general practitioners to infectious disease public health crises: an integrative systematic review of the literature. Disaster Med Public Health Prep. 2013;7(5):522-533.

3. Tan NC, Goh LG, Lee SS. Family physicians' experiences, behaviour, and use of personal protection equipment during the SARS outbreak in Singapore: do they fit the Becker Health Belief Model? Asia Pac J Public Health. 2006;18(3):49-56.

4. Wong SYS, Kung K, Wong MCS, et al. Primary care physicians' response to pandemic influenza in Hong Kong: a mixed quantitative and qualitative study. Int J Infect Dis. 2012;16(9):e687-e691.

5. Wong WC, Wong SY, Lee A, Goggins WB. How to provide an effective primary health care in fighting against severe acute respiratory syndrome: the experiences of two cities. Am J Infect Control. 2007;35(1):50-55.

6. Health Information Division, Ministry of Health, Singapore. Primary Care Survey 2014. https://www.moh.gov.sg/docs/libraries provider5/resources-statistics/reports/moh-primary-care-survey-2014report.pdf. Accessed Feb 25, 2020.

7. Chaffee M. Willingness of health care personnel to work in a disaster: an integrative review of the literature. Disaster Med Public Health Prep. 2009;3(1):42-56. 